\title{
Heart Rate n-Variability (HRnV) Measures for Prediction of Mortality in Sepsis Patients Presenting at the Emergency Department
}

\author{
Running Title: Heart Rate n-Variability (HRnV) in Sepsis
}

Nan Liu, PhD ${ }^{1,2,3 *}$, Marcel Lucas Chee ${ }^{4}$, Mabel Zhi Qi Foo ${ }^{5}$, Jeremy Zhenwen Pong, MD ${ }^{1}$, Dagang Guo, $\mathrm{PhD}^{6}$, Zhi Xiong Koh ${ }^{1,5}$, Andrew Fu Wah Ho, MBBS ${ }^{1,5}$, Chenglin Niu ${ }^{1}$, ShuLing Chong, MBBS, MPH ${ }^{1,7}$, Marcus Eng Hock Ong, MBBS, MPH ${ }^{1,5}$

${ }^{1}$ Duke-NUS Medical School, National University of Singapore, Singapore

${ }^{2}$ Health Services Research Centre, Singapore Health Services, Singapore

${ }^{3}$ Institute of Data Science, National University of Singapore, Singapore

${ }^{4}$ Faculty of Medicine, Nursing and Health Sciences, Monash University, Melbourne, Australia

${ }^{5}$ Department of Emergency Medicine, Singapore General Hospital, Singapore

${ }^{6}$ SingHealth Duke-NUS Emergency Medicine Academic Clinical Programme, Singapore

${ }^{7}$ Department of Children's Emergency, KK Women's and Chlidren's Hospital, Singapore

\section{* Corresponding Author}

Nan Liu

Centre for Quantitative Medicine and Programme in Health Services and Systems Research, Duke-NUS Medical School, 8 College Road, Singapore 169857, Singapore

Email: liu.nan@duke-nus.edu.sg

Financial Support: This work was supported by the Duke-NUS Signature Research Programme funded by the Ministry of Health, Singapore. The funder of the study had no role in study design, data collection, data analysis, data interpretation, or writing of the report.

Author Contributions: NL was responsible for study concept and design; JZP and ZXK were responsible for acquisition of the data; NL, MLC, MZQF, JZP, DG, ZXK, AFWH, CN, SLC, and MEHO were responsible for analysis and interpretation of the data; NL, MLC, MZQF, and JZP were responsible for drafting of the manuscript; NL, MLC, MZQF, JZP, DG, ZXK, AFWH, CN, SLC, and MEHO were responsible for critical revision of the manuscript for important intellectual content. NL was responsible for acquisition of funding; all authors approved the final version of the manuscript.

Conflict of Interest Disclosure: NL and MEHO are the inventors on a patent [US10299689B2] that is issued by the United States Patent and Trademark Office relevant to the material in this paper. NL, DG, ZXK, and MEHO own stock in TIIM Healthcare that produces a product relevant to the subject material. MLC, MZQF, JZP, AFWH, CN, and SLC report no conflict of interest. 


\begin{abstract}

\section{Background}

Sepsis is a potentially life threatening condition that requires prompt recognition and treatment for optimal outcomes. There is little consensus on an objective way to assess for sepsis severity and risk for mortality. In recent years, heart rate variability (HRV), a measure of the cardiac autonomic regulation derived from short electrocardiogram tracings, has been found to correlate with sepsis mortality, and its use as a prognostic variable and for risk stratification has been promising. In this paper, we present using novel heart rate n-variability $(\mathrm{HRnV})$ measures for sepsis mortality risk prediction and compare against current mortality prediction scores.
\end{abstract}

\title{
Methods
}

This study was a retrospective cohort study on a convenience sample of patients presenting to the emergency department (ED) of Singapore General Hospital between September 2014 to April 2017. Patients were included in the study if they were above 21 years old, were suspected to have sepsis by their attending physician, triaged as emergency or urgent cases, and if they met two or more of the Systemic Inflammatory Response Syndrome (SIRS) criteria. Demographic and clinical variables were obtained from the electronic medical records, and HRV and novel HRnV parameters were calculated from five minute ECG tracings. Univariable analysis was conducted on variables obtained, with the primary outcome being 30-day in-hospital mortality (IHM). Variables selected through univariable analysis and stepwise selection were included in a multivariable logistic regression model, the results of which were presented using receiver operating curve (ROC) analysis.

\section{Results}

Of 342 patients included for final analysis, $66(19 \%)$ met with the primary outcome. On univariable analysis, 85 out of 142 analysed HRV and HRnV parameters showed statistical difference between groups. The final multivariable logistic regression model comprised of 21 variables including four vital signs, two HRV parameters, and $15 \mathrm{HRnV}$ parameters. The area under the curve (AUC) of the model was 0.86 (95\% confidence interval 0.81-0.90), outperforming several established clinical scores.

\section{Conclusion}

The use of novel HRnV measures can provide adequate power to predictive models in the risk stratification of patients presenting to the ED with sepsis. When included in a multivariable logistic regression model, the HRnV-based model outperformed traditional risk stratification scoring systems. The $\mathrm{HRnV}$ measures may have potential to allow for a rapid, objective, and accurate means of patient risk stratification for sepsis severity and mortality. 


\section{Introduction}

Sepsis is a potentially life-threatening condition caused by the body's dysregulated response to infection ${ }^{1}$. Every year, more than 50 million people are affected, resulting in over five million deaths worldwide ${ }^{2}$. Prompt recognition and treatment of sepsis has been shown to impact patient outcomes, and guidelines have been developed for its management ${ }^{3}$. There is, however, a need for a rapid method to grade sepsis severity and prognosticate the risk for mortality in septic patients. A quick and accurate triage tool for risk stratification of septic patients presenting at the emergency department (ED) would thus be invaluable, allowing for greater confidence to clinical decisions, and in guiding management.

Several common disease severity scoring systems that have been utilised in the ED for the prediction of sepsis mortality including the Mortality in ED Sepsis (MEDS) score ${ }^{4}$, quick SOFA (qSOFA) ${ }^{5}$, and intensive care unit (ICU)-based scores such as the Sequential Organ Failure Assessment (SOFA) score ${ }^{6}$, and the well-established Acute Physiology and Chronic Health Evaluation II (APACHE II) score ${ }^{7}$. Although these scoring systems have shown good predictive value, certain limitations have prevented their widespread adoption ${ }^{8-11}$. In recent years, the use of heart rate variability (HRV) measurements derived from electrocardiogram (ECG) tracings have allowed for an alternative and complementary approach for the prediction of sepsis mortality. HRV analysis measures the beat-to-beat variation between each R-R interval on an ECG tracing, and is a reflection of the autonomic regulation of the cardiovascular system ${ }^{12}$. Being a non-invasive tool that can be rapidly obtained even from patients who are unable to give a history, HRV has been shown to be dysregulated in sepsis ${ }^{13}$ and correlates well with subsequent mortality ${ }^{14,15}$. Indeed, scoring systems that incorporate HRV parameters among its predictors have outperformed traditional clinical indicators and established disease severity scores in predicting sepsis mortality ${ }^{16-19}$. The use of HRV may thus further enhance our ability to stratify for risk of sepsis mortality.

In work previously conducted by our team ${ }^{20,21}$, we derived additional novel heart rate $\mathrm{n}$ variability $(\mathrm{HRnV})$ parameters with the aim of providing enhanced prognostic information to complement traditional HRV parameters. The proposed $\mathrm{HRnV}$ has two measures $-\mathrm{HR}_{n} \mathrm{~V}$ and $\mathrm{HR}_{n} \mathrm{~V}_{m} . \mathrm{HR}_{n} \mathrm{~V}$ is derived from non-overlapping R-R intervals, while $\mathrm{HR}_{n} \mathrm{~V}_{m}$ is computed from overlapping R-R intervals. For each of the traditional $\mathrm{HRV}, \mathrm{HR}_{n} \mathrm{~V}$, and $\mathrm{HR}_{n} \mathrm{~V}_{m}$ measures, time domain, frequency domain, and nonlinear analysis will yield its respective set of parameters. An application of the novel $\mathrm{HRnV}$ variables demonstrated improved predictive ability for major adverse cardiac events among patients with chest pain presenting at the $\mathrm{ED}^{21}$.

In this paper, we aim to study the prognostic ability of $\mathrm{HRnV}$ measures alongside traditional HRV parameters in predicting the outcomes in septic patients presenting at the ED, and to compare the HRnV-based model with existing mortality prediction scores.

\section{Methods}

\section{Study design and clinical setting}

We conducted a retrospective cohort analysis on a convenience sample of patients presenting to Singapore General Hospital (SGH) between September 2014 to April 2017. SGH is the largest hospital in Singapore, with its ED seeing 300 to 500 patients daily. On presentation at the ED, patients are triaged according to a symptom-based Patient Acuity Category Scale (PACS). The PACS system has four levels: PACS 1 patients are critically ill, PACS 2 patients are non-ambulant but stable, PACS 3 patients are ambulant, and PACS 4 patients are 
non-emergency. This study was approved by the SingHealth Centralized Institutional Review Board (CIRB Ref No.: 2016/2858), with requirement for patient consent waived.

\section{Study population and eligibility}

Patients were included in the study if they were aged 21 years and above, triaged to either PACS 1 or 2 at the ED, suspected to have sepsis as determined by their attending physician, and if they met two or more out of four Systemic Inflammatory Response Syndrome (SIRS) criteria ${ }^{22,23}$. The SIRS criteria (temperature $>38^{\circ} \mathrm{C}$ or $\left\langle 36^{\circ} \mathrm{C}\right.$, heart rate $>90$ beats per minute, respiratory rate $>20$ breaths per minute, and total white blood cell count $>12,000 / \mathrm{mm}^{3}$ or $<4000 / \mathrm{mm}^{3}$ ) were used despite recent revisions under the Sepsis-3 consensus that recommend for sepsis screening with qSOFA score ${ }^{1}$. This decision was made primarily to allow for comparability with the existing literature. Additionally, subsequent validation studies have disputed the utility of qSOFA over SIRS for sepsis screening in the ED due to its poor sensitivity for septic patients ${ }^{24-27}$. Patients were excluded if their ECGs had non-sinus rhythm, a high noise level ( $>30 \%$ of the entire recording), or if they had a pacemaker or were on mechanical ventilator support.

\section{Data collection}

Five-minute one-lead ECGs were performed on patients who met the inclusion criteria using the ZOLL X Series monitor/defibrillator (ZOLL Medical Corporation, Chelmsford, MA). Patient demographics, vital signs taken at triage, medical history, and laboratory investigations performed in the $\mathrm{ED}$ were retrieved from the electronic medical records. We defined the primary outcome as 30-day in-hospital mortality (IHM).

\section{HRnV representation and analysis}

Conventional HRV analysis evaluates consecutive single RR intervals (RRIs) in ECGs. We used the two novel $\mathrm{HRnV}$ measures $\left(\mathrm{HR}_{n} \mathrm{~V}\right.$ and $\left.\mathrm{HR}_{n} \mathrm{~V}_{m}\right)$ to represent beat-to-beat variations in $\mathrm{ECG}^{20,21}$, and have provided a visual illustration in Figure 1.

To define the $\mathrm{HR}_{n} \mathrm{~V}$ measure, a new type of RRI called $\mathrm{RR}_{n} \mathrm{I}$ is obtained, where $n$ is an integer between 1 and $N$ and $N$ (number of conventional RRIs combined to form a new $\mathrm{RR}_{n} \mathrm{I}$ ) is much smaller than $N \square$ (total number of RRIs). With newly generated $\mathrm{RR}_{n} \mathrm{I}$ sequences, traditional time and frequency domains, and nonlinear analyses ${ }^{28,29}$ are applied to calculate $\mathrm{HR}_{n} \mathrm{~V}$ parameters. In addition to conventional $\mathrm{HRV}$ parameters, $\mathrm{HR}_{n} \mathrm{~V}$ also evaluates two newly created parameters: NN50n and pNN50n. These two parameters differ from the traditional NN50 and pNN50 parameters in that the threshold is changed from $50 \mathrm{~ms}$ to $50 \times n$ ms in describing the absolute difference between successive $\mathrm{RR}_{n} \mathrm{Is}$.

Similarly, $\mathrm{HR}_{n} \mathrm{~V}_{m}$ is a measure derived from $\mathrm{RR}_{n} \mathrm{I}_{m}$, where $m$ is the number determining nonoverlapping RRIs for each $\mathrm{RR}_{n} \mathrm{I}$. When $m=n, \mathrm{RR}_{n} \mathrm{I}_{m}$ becomes $\mathrm{RR}_{n} \mathrm{I}$ as there are no overlapping RRIs, resulting in an upper limit of $N-1$ for $m$. Utilising all permissible combinations of $n$ and $m, N(N+1) / 2$ sets of traditional $\mathrm{HRV}$, novel $\mathrm{HR}_{n} \mathrm{~V}$ and $\mathrm{HR}_{n} \mathrm{~V}_{m}$ parameters can be generated from a single RRI sequence. In our analysis, we set the upper limit of $N$ as 3, due to the relatively short duration of collected ECG samples. As a result, one set of HRV parameters, two sets of $\mathrm{HR}_{n} \mathrm{~V}\left(\mathrm{HR}_{2} \mathrm{~V}\right.$ and $\left.\mathrm{HR}_{3} \mathrm{~V}\right)$ parameters, and three sets of $\mathrm{HR}_{n} \mathrm{~V}_{m}\left(\mathrm{HR}_{2} \mathrm{~V}_{1}, \mathrm{HR}_{3} \mathrm{~V}_{1}\right.$, and $\left.\mathrm{HR}_{3} \mathrm{~V}_{2}\right)$ parameters can be calculated. The HRnV-Calc software suite (https://github.com/nliulab/HRnV) was used for calculating the HRV and HRnV parameters, in which the functions from PhysioNet Cardiovascular Signal Toolbox ${ }^{30}$ were performed for ECG signal processing. 


\section{Statistical analysis}

Categorical variables were compared between patients who did and did not meet the primary outcome (30-day IHM) using $\chi^{2}$ test or Fisher's exact test where appropriate. Continuous variables were checked for normality with the Kolmogorov-Smirnov test. Subsequently, normally distributed variables were presented as mean and standard deviation (SD) and were compared with independent two-tailed $t$ test between groups, while non-normally distributed variables were presented as median and interquartile range (IQR; $25^{\text {th }}$ to $75^{\text {th }}$ percentiles) and compared using the Mann-Whitney U test.

Univariable regression analysis was conducted on traditional HRV parameters, novel HRnV parameters and demographic and clinical variables. Each variable was evaluated as an individual predictor of the primary outcome (30-day IHM) using binary logistic regression with odds ratio (OR), 95\% confidence interval (CI) and p-value reported. For multivariable regression analysis, we adjusted for age, temperature, systolic blood pressure, heart rate, and Glasgow Coma Scale (GCS) as these variables were either shown to be significant predictors of sepsis mortality in previous literature ${ }^{15,31-33}$, or are included in well-established sepsis scoring systems such as the National Early Warning Score (NEWS $)^{34}$, Modified Early Warning Score (MEWS) ${ }^{35}$, MEDS, qSOFA, or APACHE II. HRV and HRnV parameters were included in the multivariable analysis if they achieved $\mathrm{p}<0.2$ in the univariable analysis. Included variables were then checked for collinearity using Pearson's R correlation. For each collinear pair, the variable with the higher p-value on univariate analysis was eliminated until no collinear pairs remained. The remaining variables were then fed into a backward stepwise multivariable logistic regression model which used $\mathrm{p}<0.1$ as an endpoint. We took statistical significance at $p$-value $<0.05$. A receiver operating characteristic (ROC) curve was plotted to assess the predictive ability of the multivariable regression model and compared against other established disease scoring systems on their area under the curve (AUC).

Missing data were addressed by median imputation, in consideration of the low proportion of missing data $(<0.3 \%)$ for each variable, the nature of variables, and recommendations for missing data in clinical trials ${ }^{36}$. There were three missing observations for which the median value was imputed; one patient had unknown medical history of cancer, and another patient was missing both initial and worst qSOFA scores.

All statistical analyses were carried out using Python version 3.8.0 (Python Software Foundation, Delaware, USA) using the SciPy library (version 1.3.1). Regression models were built using the StatsModels library (version 0.10.2) and scikit-learn library (version 0.22).

\section{Results}

\section{Patient recruitment}

Figure 2 presents the patient recruitment flowchart. Of the 659 patients that were initially recruited, 190 patients did not meet the SIRS criteria, and 127 patients had inapplicable ECG readings. A total of 342 patients were included for analysis and classified depending on whether they met the primary outcome of 30-day IHM $(n=66,19 \%)$, or did not meet the primary outcome $(\mathrm{n}=276,81 \%)$.

\section{Baseline characteristics and clinical parameters}

Table 1 illustrates baseline characteristics and clinical parameters of patients who met and did not meet with 30-day IHM. Patients who met with 30-day IHM were older and presented with higher respiratory rates but lower temperatures, systolic blood pressures (SBP) and 
Glagow Coma Scale (GCS) scores, when compared to patients who did not meet with 30-day IHM. The worst recorded values of respiratory rate, GCS, and SBP during each patient's ED stay were also significantly more abnormal in patients that met with 30-day IHM. Difference in disposition from the ED was significant, with a larger proportion of patients who eventually met with 30-day IHM requiring admission to the ICU as compared to patients who did not meet with 30 -day IHM (16.7\% vs 4.3\%, p=0.001). Additionally, a larger proportion of patients who met with 30-day IHM had a respiratory source of infection (45.5\% vs $27.2 \%$, $\mathrm{p}=0.006$ ) while a smaller proportion had a source of infection originating from the urinary tract $(7.6 \%$ vs $25.7 \%, \mathrm{p}=0.003$ ) when compared to patients who did not meet with 30-day IHM. No significant differences were detected in gender, PACS status, ethnicity, or medical history between both groups.

\section{HRV and HRnV parameter description and univariable analysis}

Table 2 presents the descriptive analysis of HRV and HRnV parameters. In this study, $N$ was set as 3 and $\mathrm{HR}_{2} \mathrm{~V}, \mathrm{HR}_{2} \mathrm{~V}_{1}, \mathrm{HR}_{3} \mathrm{~V}, \mathrm{HR}_{3} \mathrm{~V}_{1}$ and $\mathrm{HR}_{3} \mathrm{~V}_{2}$ parameters were calculated. Among time domain parameters such as mean $\mathrm{NN}$ and $\mathrm{SDNN}, \mathrm{HR}_{n} \mathrm{~V}$ and $\mathrm{HR}_{n} \mathrm{~V}_{m}$ values are generally directly proportional to $n$ and increase when $n$ increases. $\mathrm{HR}_{2} \mathrm{~V}$ SampEn and $\mathrm{HR}_{3} \mathrm{~V}$ SampEn were considerably larger compared to SampEn parameters of $\mathrm{HRV}, \mathrm{HR}_{2} \mathrm{~V}_{1}, \mathrm{HR}_{3} \mathrm{~V}_{1}$, and $\mathrm{HR}_{3} \mathrm{~V}_{2}$. This was due to insufficient data points since our ECG recordings were only five minutes long. $\mathrm{HR}_{2} \mathrm{~V}_{1}, \mathrm{HR}_{3} \mathrm{~V}_{1}$ and $\mathrm{HR}_{3} \mathrm{~V}_{2}$ did not encounter this limitation as more data points were available from calculation using overlapping $\mathrm{RR}_{n} \mathrm{I}_{m}$ sequences ${ }^{21}$.

Table 3 shows the results of univariable analysis of HRV and HRnV parameters. Of 142 HRV and HRnV parameters, 85 were significantly different between the two outcome groups. Specifically, $14 \mathrm{HRV}, 14 \mathrm{HR}_{2} \mathrm{~V}, 16 \mathrm{HR}_{2} \mathrm{~V}_{1}, 11 \mathrm{HR}_{3} \mathrm{~V}, 16 \mathrm{HR}_{3} \mathrm{~V}_{1}$, and $14 \mathrm{HR}_{3} \mathrm{~V}_{2}$ parameters were statistically significant. In at least four out of six HRnV measures, RMSSD, kurtosis, NN50, pNN50, NN50n, pNN50n, HF power, HF power norm, Poincare SD1, and Poincare SD1/SD2 were significantly higher, while LF power norm and DFA $\alpha 2$ were significantly lower in patients who met the primary outcome compared to those who did not. Additionally, VLF power and DFA $\alpha 1$ were not significant in HRV analysis but were statistically significant in several HRnV measures.

Overall, six baseline characteristics (age and vital signs at triage including temperature, respiratory rate, $\mathrm{SpO}_{2}, \mathrm{SBP}$ and GCS), $17 \mathrm{HRV}$ parameters, and $96 \mathrm{HRnV}$ parameters had $\mathrm{p}<0.2$ on univariable analysis. After collinearity assessment, the remaining 87 variables were entered into a stepwise-selection regression model.

\section{Multivariable analysis and ROC analysis}

Table 4 presents the multivariable analysis of variables found to be significantly different on univariable analysis. A total of 21 out of 87 variables were selected through stepwise selection. Of the 21 variables, 16 showed $\mathrm{p}<0.05$. These include vital signs such as respiratory rate $(\mathrm{OR}=1.168 ; 95 \% \mathrm{CI} 1.085-1.257$; $\mathrm{p}<0.001)$, SBP $(\mathrm{OR}=0.978$; 95\% CI 0.966$0.990 ; \mathrm{p}=0.001), \mathrm{SpO}_{2}(\mathrm{OR}=0.892 ; 95 \% \mathrm{CI} 0.838-0.950 ; \mathrm{p}=<0.001)$, and GCS $(\mathrm{OR}=0.845$; 95\% CI 0.769-0.929; $\mathrm{p}=0.001)$, and $\mathrm{HRnV}$ measures such as $\mathrm{HR}_{2} \mathrm{~V}_{1} \mathrm{NN50}(\mathrm{OR}=0.808 ; 95 \%$ CI 0.682-0.958; $\mathrm{p}=0.014), \mathrm{HR}_{2} \mathrm{~V}$ pNN50 (OR=0.290; 95\% CI 0.115-0.732; $\left.\mathrm{p}=0.009\right)$, $\mathrm{HR}_{2} \mathrm{~V}_{1}$ pNN50 (OR=5.700; 95\% CI 1.784-18.213; $\left.\mathrm{p}=0.003\right), \mathrm{HR}_{2} \mathrm{~V}$ ApEn (OR=0.106; 95\% CI 0.013-0.877; $\mathrm{p}=0.037$ ) and several $\mathrm{HR}_{3} \mathrm{~V}_{1}$ and $\mathrm{HR}_{3} \mathrm{~V}_{2}$ parameters which demonstrated strong predictive power in assessing the risk for 30-day IHM. The final multivariable predictive model consisted of four vital signs, two traditional HRV parameters, and 15 novel HRnV parameters. Hereafter, we refer to this model as the HRnV model. 
A ROC curve was plotted for assessment of the HRnV model and compared against established disease severity scoring systems for the prediction of 30-day IHM in patients presenting to the ED with sepsis (Figure 3). The AUC of the HRnV model was 0.86 (95\% CI: 0.81-0.90), outperforming the AUC of NEWS 0.71 (95\% CI: 0.64-0.78), MEWS 0.60 (95\% CI: 0.53-0.67), MEDS 0.85 (95\% CI: 0.81-0.90), SOFA 0.71 (95\% CI: 0.64-0.78), APACHE II 0.74 (95\% CI: 0.68-0.80), and the patient's worst qSOFA value 0.72 (95\% CI: 0.65-0.79).

\section{Discussion}

In recent years, there has been a surge in research interest in HRV and its ability to prognosticate for adverse patient outcomes across various disease processes ${ }^{18,19,29}$. To improve the predictive ability of HRV, several studies have sought to utilise advanced nonlinear techniques to derive novel HRV parameters ${ }^{30,31}$. Indeed, we previously employed the above novel HRnV measures to assess for risk of 30-day major adverse cardiac events (MACE) in patients presenting to the ED with chest pain to good effect ${ }^{21}$.

In this study, we evaluated the predictive value of novel $\mathrm{HRnV}$ measures $\left(\mathrm{HR}_{n} \mathrm{~V}\right.$ and $\left.\mathrm{HR}_{n} \mathrm{~V}_{m}\right)$ in assessing the risk of 30-day IHM in patients presenting to the ED with sepsis. In addition to the 22 traditional HRV parameters, we derived an additional $120 \mathrm{HRnV}$ parameters, 71 of which were found to be statistically significant in their association with the primary outcome. The newly generated HRnV parameters greatly amplify the number of candidate predictors, and have demonstrated improved predictive ability for sepsis mortality. The newly added parameters, NN50n and pNN50n, are significantly associated with mortality in the univariate analysis, and characterise the number of times that the absolute difference between two successive $\mathrm{RR}_{n} \mathrm{I}$ sequences exceeds $50 \times n \mathrm{~ms}$, by assuming the absolute difference may be magnified when the corresponding $\mathrm{RR}_{n} \mathrm{I}$ is $n$ times longer than $\mathrm{RRI}^{21}$. The composite HRV$\mathrm{HRnV}$ model as derived from multivariable logistic regression achieved the highest AUC on ROC analysis, and outperformed other established disease scoring systems such as NEWS, MEWS, MEDS, SOFA, and APACHE II for the prediction of 30-day IHM in patients presenting to the ED with sepsis.

In addition to demonstrating superior predictive ability for sepsis mortality, the HRnV model is made even more relevant in its ability for rapid and objective prognostication where only vital signs and parameters calculated from five-minute ECG tracings are needed. Many established disease severity scores require invasive tests which require long turnaround times and resources to obtain or include subjective parameters that involve interrater variability while scoring. Among disease severity scores with higher AUCs, the MEDS score, developed specifically for the purpose of risk stratification of septic patients in the ED, suffers from some of these limitations and its adoption has thus not been widespread. APACHE II and SOFA scores initially designed for use in the intensive care unit (ICU) setting similarly requires invasive investigations in the calculation of its score. In these aspects, the HRnV model which is derived from vital signs taken on ED presentation, and HRV and HRnV measures calculated from a five minute ECG tracing, can overcome these limitations, and provide a rapid, objective, and accurate risk assessment of the septic patient. A triage tool with these characteristics would be invaluable to the physician and can aid in risk stratification, clinical management, patient disposition, and accurate patient classification for administrative or research purposes. 
Limitations: Despite the presentation of many HRnV parameters in this study, there exist technical limitations which constrain their utility. First, a majority of the HRnV variables were removed from predictive modelling with traditional logistic regression, which hindered the release of the power of the novel HRnV representation. Moving forward, we endeavour to explore the use of machine learning algorithms ${ }^{37-39}$ for full utilization of the $\mathrm{HRnV}$ parameters. Second, the difficulty in interpretating the HRnV parameters may pose a challenge in their clinical implementation and adoption. Third, although the novel HRnV variables and corresponding model have shown strong predictive value and potential clinical utility, this study was a single-center, retrospective observational study, thus multi-center trials would be required to corroborate our results. Prospective cohort studies would also be valuable in validating the effectiveness of the HRnV parameters, and more conclusively establishing a cause and effect relationship.

\section{Conclusions}

The use of novel HRV measures $\left(\mathrm{HR}_{n} \mathrm{~V}\right.$ and $\left.\mathrm{HR}_{n} \mathrm{~V}_{m}\right)$ can provide additional power to predictive models in the risk stratification of patients who present to the ED with sepsis. When included in a model with other clinical variables, the HRnV model outperforms traditional risk stratification scoring systems. The use of HRnV measures may allow for a rapid, objective, and accurate means of patient risk stratification for sepsis severity and mortality.

\section{References}

1. Singer M, Deutschman CS, Seymour CW, et al. The Third International Consensus Definitions for Sepsis and Septic Shock (Sepsis-3). Jama 2016;315:801-10.

2. Fleischmann C, Scherag A, Adhikari NK, et al. Assessment of Global Incidence and Mortality of Hospital-treated Sepsis. Current Estimates and Limitations. Am J Respir Crit Care Med 2016;193:259-72.

3. Rhodes A, Evans LE, Alhazzani W, et al. Surviving Sepsis Campaign: International Guidelines for Management of Sepsis and Septic Shock: 2016. Intensive Care Med 2017;43:304-77.

4. Shapiro NI, Wolfe RE, Moore RB, Smith E, Burdick E, Bates DW. Mortality in Emergency Department Sepsis (MEDS) score: a prospectively derived and validated clinical prediction rule. Crit Care Med 2003;31:670-5.

5. Seymour CW, Liu VX, Iwashyna TJ, et al. Assessment of Clinical Criteria for Sepsis: For the Third International Consensus Definitions for Sepsis and Septic Shock (Sepsis-3). JAMA 2016;315:762-74.

6. Vincent JL, Moreno R, Takala J, et al. The SOFA (Sepsis-related Organ Failure Assessment) score to describe organ dysfunction/failure. On behalf of the Working Group on Sepsis-Related Problems of the European Society of Intensive Care Medicine. Intensive Care Med 1996;22:707-10.

7. Knaus WA, Draper EA, Wagner DP, Zimmerman JE. APACHE II: A severity of disease classification system. Crit Care Med 1985;13:818-29.

8. Macdonald SP, Arendts G, Fatovich DM, Brown SG. Comparison of PIRO, SOFA, and MEDS scores for predicting mortality in emergency department patients with severe sepsis and septic shock. Acad Emerg Med 2014;21:1257-63.

9. Williams JM, Greenslade JH, Chu K, Brown AF, Lipman J. Severity Scores in Emergency Department Patients With Presumed Infection: A Prospective Validation Study. Crit Care Med 2016;44:539-47. 
10. Hilderink MJ, Roest AA, Hermans M, Keulemans YC, Stehouwer CD, Stassen PM. Predictive accuracy and feasibility of risk stratification scores for 28-day mortality of patients with sepsis in an emergency department. European journal of emergency medicine : official journal of the European Society for Emergency Medicine 2015;22:331-7.

11. Pong JZ, Koh ZX, Samsudin MI, Fook-Chong S, Liu N, Ong MEH. Validation of the mortality in emergency department sepsis (MEDS) score in a Singaporean cohort. Medicine (Baltimore) 2019;98:e16962.

12. Malik M, Camm AJ, Bigger Jr JT, et al. Heart rate variability. Standards of measurement, physiological interpretation, and clinical use. European Heart Journal 1996; 17:354-81.

13. Scheff JD, Griffel B, Corbett SA, Calvano SE, Androulakis IP. On heart rate variability and autonomic activity in homeostasis and in systemic inflammation.

Mathematical biosciences 2014;252:36-44.

14. Barnaby D, Ferrick K, Kaplan DT, Shah S, Bijur P, Gallagher EJ. Heart rate variability in emergency department patients with sepsis Acad Emerg Med 2002;9:661 -70. 15. Chen WL, Chen JH, Huang CC, Kuo CD, Huang CI, Lee LS. Heart rate variability measures as predictors of in-hospital mortality in ED patients with sepsis. Am J Emerg Med 2008;26:395-401.

16. Pong JZ, Fook-Chong S, Koh ZX, et al. Combining Heart Rate Variability with Disease Severity Score Variables for Mortality Risk Stratification in Septic Patients Presenting at the Emergency Department. Int J Environ Res Public Health 2019;16. 17. de Castilho FM, Ribeiro ALP, da Silva JLP, Nobre V, de Sousa MR. Heart rate variability as predictor of mortality in sepsis: A prospective cohort study. PLoS One 2017; 12:e0180060.

18. Barnaby DP, Fernando SM, Herry CL, Scales NB, Gallagher EJ, Seely AJE. Heart Rate Variability, Clinical and Laboratory Measures to Predict Future Deterioration in Patients Presenting With Sepsis. Shock 2019;51:416-22.

19. Arnold R, Green G, Bravi A, Hollenberg S, Seely A. Impaired heart rate variability predicts clinical deterioration and progressive organ failure in emergency department sepsis patients. Crit Care 2012;16:P37-P.

20. Liu N, Guo D, Koh ZX, Ho AFW, Ong MEH. Heart rate n-variability (HRnV): A novel representation of beat-to-beat variation in electrocardiogram. bioRxiv 2018:449504; doi: https://doi.org/10.1101/.

21. Liu N, Guo D, Koh ZX, et al. Heart rate n-variability (HRnV) and its application to risk stratification of chest pain patients in the emergency department. BMC Cardiovasc Disord 2020;20:168.

22. Bone RC, Balk RA, Cerra FB, et al. Definitions for sepsis and organ failure and guidelines for the use of innovative therapies in sepsis. The ACCP/SCCM Consensus Conference Committee. American College of Chest Physicians/Society of Critical Care Medicine. Chest 1992;101:1644-55.

23. Levy MM, Fink MP, Marshall JC, et al. 2001 SCCM/ESICM/ACCP/ATS/SIS International Sepsis Definitions Conference. Crit Care Med 2003;31:1250-6.

24. Simpson SQ. New Sepsis Criteria. Chest 2016;149:1117-8.

25. Fernando SM, Tran A, Taljaard M, et al. Prognostic Accuracy of the Quick Sequential Organ Failure Assessment for Mortality in Patients With Suspected Infection. Annals of Internal Medicine 2018;168.

26. Van der Woude SWvD, F.F.; Hutten, B.A.; Nellen, F.J.; Holleman, F. Classifying sepsis patients in the emergency department using SIRS, qSOFA or MEWS. Neth J Med 2018;76:158-66. 
medRxiv preprint doi: https://doi.org/10.1101/2020.12.26.20248866; this version posted January 2, 2021. The copyright holder for this preprint (which was not certified by peer review) is the author/funder, who has granted medRxiv a license to display the preprint in perpetuity.

It is made available under a CC-BY-NC-ND 4.0 International license.

27. Usman OA, Usman AA, Ward MA. Comparison of SIRS, qSOFA, and NEWS for the early identification of sepsis in the Emergency Department. Am J Emerg Med 2019;37:14907.

28. Electrophysiology TFotES. Heart Rate Variability. Circulation 1996;93:1043-65.

29. Shaffer F, Ginsberg JP. An Overview of Heart Rate Variability Metrics and Norms. Front Public Health 2017;5:258.

30. Vest AN, Da Poian G, Li Q, et al. An open source benchmarked toolbox for cardiovascular waveform and interval analysis. Physiol Meas 2018;39:105004.

31. Lopez-Delgado JC, Brink A, Alsma J, et al. Predicting mortality in patients with suspected sepsis at the Emergency Department; A retrospective cohort study comparing qSOFA, SIRS and National Early Warning Score. Plos One 2019;14.

32. Samsudin MuI, Liu N, Prabhakar SM, et al. A novel heart rate variability based risk prediction model for septic patients presenting to the emergency department. Medicine $2018 ; 97$.

33. Sanderson M, Chikhani M, Blyth E, et al. Predicting 30-day mortality in patients with sepsis: An exploratory analysis of process of care and patient characteristics. Journal of the Intensive Care Society 2018;19:299-304.

34. Smith GB, Prytherch DR, Meredith P, Schmidt PE, Featherstone PI. The ability of the National Early Warning Score (NEWS) to discriminate patients at risk of early cardiac arrest, unanticipated intensive care unit admission, and death. Resuscitation 2013;84:465-70.

35. Subbe CP. Validation of a modified Early Warning Score in medical admissions. Qjm 2001;94:521-6.

36. Little RJ, D'Agostino R, Cohen ML, et al. The Prevention and Treatment of Missing Data in Clinical Trials. New England Journal of Medicine 2012;367:1355-60.

37. Chiew CJ, Liu N, Tagami T, Wong TH, Koh ZX, Ong MEH. Heart rate variability based machine learning models for risk prediction of suspected sepsis patients in the emergency department. Medicine 2019;98:e14197.

38. Ross EG, Shah NH, Dalman RL, Nead KT, Cooke JP, Leeper NJ. The use of machine learning for the identification of peripheral artery disease and future mortality risk. J Vasc Surg 2016;64:1515-22.e3.

39. Xie F, Chakraborty B, Ong MEH, Goldstein BA, Liu N. AutoScore: A Machine Learning-Based Automatic Clinical Score Generator and Its Application to Mortality Prediction Using Electronic Health Records. JMIR medical informatics 2020:21798. 


\section{Tables}

Table 1. Baseline characteristics and clinical parameters

\begin{tabular}{|c|c|c|c|}
\hline Variable & $\begin{array}{l}\text { No 30-day IHM } \\
(n=276)\end{array}$ & $\begin{array}{l}\text { 30-day IHM } \\
(\mathrm{n}=66)\end{array}$ & $P$-value \\
\hline Age, mean (SD) & $65.8(16.1)$ & $73.2(14.8)$ & $0.001 *$ \\
\hline Male gender, $\mathrm{n}(\%)$ & $144.0(52.2)$ & $30.0(45.5)$ & 0.399 \\
\hline Triaged to high acuity (PACS1), n (\%) & $254.0(92.0)$ & $64.0(97.0)$ & 0.19 \\
\hline SIRS criteria met, $\mathrm{n}(\%)$ & 250.0 (90.6) & $58.0(87.9)$ & 0.667 \\
\hline Race, $\mathrm{n}(\%)$ & & & 0.946 \\
\hline Chinese & $203.0(73.6)$ & $49.0(74.2)$ & 0.967 \\
\hline Malay & $40.0(14.5)$ & $8.0(12.1)$ & 0.763 \\
\hline Indian & $21.0(7.6)$ & $6.0(9.1)$ & 0.883 \\
\hline Other & $12.0(4.3)$ & $3.0(4.5)$ & 1 \\
\hline Disposition from ED, n (\%) & & & $0.001 *$ \\
\hline Intensive care unit & $12.0(4.3)$ & $11.0(16.7)$ & $0.001 *$ \\
\hline high-dependency unit & $28.0(10.1)$ & $3.0(4.5)$ & 0.231 \\
\hline General ward & $236.0(85.5)$ & $52.0(78.8)$ & 0.247 \\
\hline \multicolumn{4}{|l|}{ Medical history, n (\%) } \\
\hline Ischemic heart disease & $73.0(26.4)$ & $21.0(31.8)$ & 0.469 \\
\hline Diabetes & $111.0(40.2)$ & $24.0(36.4)$ & 0.663 \\
\hline Hypertension & $156.0(56.5)$ & $35.0(53.0)$ & 0.708 \\
\hline Cancer & $79.0(28.6)$ & $23.0(34.8)$ & 0.399 \\
\hline Serious infection & $117.0(42.4)$ & $28.0(42.4)$ & 0.894 \\
\hline \multicolumn{4}{|l|}{ Source of infection, n (\%) } \\
\hline Respiratory & $75.0(27.2)$ & $30.0(45.5)$ & $0.006^{*}$ \\
\hline Urinary tract & $71.0(25.7)$ & $5.0(7.6)$ & $0.003 *$ \\
\hline Gastrointestinal & $18.0(6.5)$ & $4.0(6.1)$ & 1 \\
\hline Musculoskeletal & $11.0(4.0)$ & $3.0(4.5)$ & 0.738 \\
\hline Hepatobiliary & $20.0(7.2)$ & $0.0(0.0)$ & $0.018^{*}$ \\
\hline Peritoneum & $3.0(1.1)$ & $2.0(3.0)$ & 0.248 \\
\hline Skin & $3.0(1.1)$ & $0.0(0.0)$ & 1 \\
\hline Line & $7.0(2.5)$ & $0.0(0.0)$ & 0.354 \\
\hline Cardiac & $7.0(2.5)$ & $2.0(3.0)$ & 0.686 \\
\hline Central nervous system & $1.0(0.4)$ & $0.0(0.0)$ & 1 \\
\hline Unknown & $23.0(8.3)$ & $12.0(18.2)$ & $0.032 *$ \\
\hline No infection & $37.0(13.4)$ & $8.0(12.1)$ & 0.94 \\
\hline \multicolumn{4}{|c|}{ Vital sign predictors, mean (SD) or median (IQR) } \\
\hline Heart rate, bpm & $114.2(23.3)$ & $112.7(26.0)$ & 0.652 \\
\hline White blood cell count & $14.0(7.7)$ & $13.0(9.6)$ & 0.36 \\
\hline Diastolic BP, mmHg & $63.0(19.5)$ & $59.7(17.4)$ & 0.213 \\
\hline Temperature, $\square$ & $38.1(37.1-38.8)$ & $37.2(36.3-38.0)$ & $<0.001 *$ \\
\hline Respiratory rate, bpm & $19.0(18.0-21.0)$ & $22.0(19.0-25.0)$ & $<0.001 *$ \\
\hline Respiratory rate (worst), bpm & $21.0(19.8-24.0)$ & $26.0(22.0-30.0)$ & $<0.001 *$ \\
\hline Systolic BP, mmHg & $109.0(86.0-139.0)$ & $\begin{array}{l}101.0(78.0- \\
118.5)\end{array}$ & $0.012 *$ \\
\hline Systolic BP (worst), mmHg & $90.0(77.0-109.2)$ & $78.0(63.2-94.8)$ & $<0.001 *$ \\
\hline GCS (3-15) & $13.4(3.0)$ & $11.7(4.1)$ & $0.001 *$ \\
\hline
\end{tabular}


medRxiv preprint doi: https://doi.org/10.1101/2020.12.26.20248866; this version posted January 2, 2021. The copyright holder for this preprint (which was not certified by peer review) is the author/funder, who has granted medRxiv a license to display the preprint in perpetuity.

\begin{abstract}
It is made available under a CC-BY-NC-ND 4.0 International license.
\end{abstract}

\begin{tabular}{|c|c|c|c|}
\hline Worst GCS (3-15) & $13.4(3.1)$ & $11.7(4.1)$ & 0.00 \\
\hline
\end{tabular}


medRxiv preprint doi: https://doi.org/10.1101/2020.12.26.20248866; this version posted January 2, 2021. The copyright holder for this preprint (which was not certified by peer review) is the author/funder, who has granted medRxiv a license to display the preprint in perpetuity.

It is made available under a CC-BY-NC-ND 4.0 International license.

Table 2. Descriptive analyses of heart rate variability (HRV) and heart rate n-variability $(\mathrm{HRnV})$ parameters

\begin{tabular}{|c|c|c|c|c|c|c|}
\hline & HRV & $\mathrm{HR}_{2} \mathrm{~V}$ & $\mathrm{HR}_{2} \mathrm{~V}_{1}$ & $\mathrm{HR}_{3} \mathrm{~V}$ & $\mathrm{HR}_{3} \mathrm{~V}_{1}$ & $\mathrm{HR}_{3} \mathrm{~V}_{2}$ \\
\hline Mean NN (ms) & $577.32(135.86)$ & $1150.61(270.41)$ & $1154.63(271.70)$ & $1723.85(406.01)$ & $1731.93(407.53)$ & $1725.94(405.63)$ \\
\hline SDNN (ms) & $22.72(20.80)$ & $28.90(25.18)$ & $34.55(36.99)$ & $35.99(33.28)$ & $46.02(53.18)$ & $37.39(34.43)$ \\
\hline Mean HR (bpm) & $109.20(22.75)$ & $54.71(11.38)$ & $54.55(11.37)$ & $36.51(7.60)$ & $36.36(7.58)$ & $36.46(7.58)$ \\
\hline SD HR (bpm) & $4.28(3.10)$ & $1.38(1.14)$ & $1.56(1.22)$ & $0.75(0.66)$ & $0.91(0.74)$ & $0.78(0.67)$ \\
\hline RMSSD (ms) & $26.65(21.38)$ & $24.03(19.70)$ & $24.94(20.91)$ & $24.75(20.61)$ & $24.97(20.76)$ & $24.17(19.72)$ \\
\hline Skewness & $0.02(3.33)$ & $-0.21(2.25)$ & $-0.11(2.61)$ & $-0.16(2.09)$ & $-0.10(2.10)$ & $-0.16(1.97)$ \\
\hline Kurtosis & $24.80(62.04)$ & $12.36(28.30)$ & $16.65(38.92)$ & $10.03(22.30)$ & $12.08(25.82)$ & $10.02(22.53)$ \\
\hline Triangular index & $4.47(3.17)$ & $5.92(4.16)$ & $6.78(4.54)$ & $6.75(4.16)$ & $8.77(5.94)$ & $7.29(4.73)$ \\
\hline NN50 (count) & $44.08(72.93)$ & $18.34(34.43)$ & $36.86(69.62)$ & $12.51(23.53)$ & $37.31(69.48)$ & $18.85(35.29)$ \\
\hline pNN50 (\%) & $7.56(13.29)$ & $6.43(12.67)$ & $6.46(12.79)$ & $6.65(13.18)$ & $6.60(12.90)$ & $6.67(13.17)$ \\
\hline NN50n (count) & - & $1.54(5.85)$ & $3.33(12.00)$ & $1.05(3.66)$ & $3.34(10.97)$ & $1.50(5.23)$ \\
\hline pNN50n (\%) & - & $0.64(2.69)$ & $0.69(2.74)$ & $0.64(2.37)$ & $0.69(2.42)$ & $0.62(2.29)$ \\
\hline Total power $\left(\mathrm{ms}^{2}\right)$ & $911.66(2856.13)$ & $\begin{array}{l}1224.37 \\
(2983.21) \\
\end{array}$ & $\begin{array}{l}3189.41 \\
(10678.24)\end{array}$ & $\begin{array}{l}1563.63 \\
(3803.04) \\
\end{array}$ & $\begin{array}{l}6532.50 \\
(23296.45)\end{array}$ & $\begin{array}{l}2121.76 \\
(5730.04)\end{array}$ \\
\hline VLF power $\left(\mathrm{ms}^{2}\right)$ & $285.42(1302.27)$ & $441.08(1533.75)$ & $\begin{array}{l}1135.39 \\
(5206.55)\end{array}$ & $654.27(2024.13)$ & $\begin{array}{l}2545.77 \\
(11693.83)\end{array}$ & $976.18(3417.38)$ \\
\hline LF power $\left(\mathrm{ms}^{2}\right)$ & $188.81(612.89)$ & 338.39 (915.03) & $706.26(2355.90)$ & $357.25(862.83)$ & $\begin{array}{l}1496.80 \\
(5051.87)\end{array}$ & $605.97(1723.07)$ \\
\hline HF power $\left(\mathrm{ms}^{2}\right)$ & $256.53(719.39)$ & $316.90(617.51)$ & 631.87 (1365.09) & $345.05(986.15)$ & $892.93(1977.84)$ & $252.99(488.08)$ \\
\hline LF power norm $(n u)$ & $36.81(18.49)$ & $42.85(17.77)$ & $42.43(18.63)$ & $56.43(17.98)$ & $51.19(18.96)$ & $61.24(16.92)$ \\
\hline HF power norm (nu) & $63.19(18.49)$ & $57.15(17.77)$ & $57.57(18.63)$ & $43.57(17.98)$ & $48.81(18.96)$ & $38.76(16.92)$ \\
\hline LF/HF & $0.86(1.11)$ & $1.00(0.93)$ & $1.11(1.43)$ & $1.83(1.75)$ & $1.72(2.35)$ & $2.31(2.10)$ \\
\hline Poincaré SD1 (ms) & $18.63(14.60)$ & $17.08(14.23)$ & $17.38(14.09)$ & $18.13(16.08)$ & $17.45(14.19)$ & $17.23(14.24)$ \\
\hline Poincaré SD2 (ms) & $24.51(25.84)$ & $35.89(32.43)$ & $44.16(49.92)$ & $44.12(46.37)$ & $61.00(73.15)$ & 48.15 (45.59) \\
\hline Poincaré SD1/SD2 ratio & $0.87(0.38)$ & $0.52(0.21)$ & $0.46(0.20)$ & $0.47(0.22)$ & $0.35(0.19)$ & $0.42(0.21)$ \\
\hline SampEn & $1.35(0.46)$ & 384.67 (5004.12) & $1.41(0.48)$ & $767.92(7056.05)$ & $1.38(0.52)$ & $384.66(5004.12)$ \\
\hline ApEn & $1.16(0.22)$ & $0.93(0.22)$ & $1.13(0.22)$ & $0.70(0.23)$ & $1.10(0.24)$ & $0.90(0.23)$ \\
\hline DFA, $\alpha 1$ & $0.57(0.26)$ & $0.83(0.28)$ & $0.85(0.28)$ & $1.04(0.30)$ & $1.09(0.28)$ & $1.07(0.30)$ \\
\hline DFA, $\alpha 2$ & $0.83(0.28)$ & $0.81(0.39)$ & $0.90(0.27)$ & $0.55(0.70)$ & $0.95(0.26)$ & $0.87(0.39)$ \\
\hline
\end{tabular}

HRV: heart rate variability; mean NN: average of R-R intervals; SDNN: standard deviation of R-R intervals; RMSSD: square root of the mean squared differences between R-R intervals; NN50: the number of times that the absolute difference between two successive R-R intervals exceeds 50 ms; pNN50: NN50 divided by the total number of R-R intervals; NN50n: the number of times that the absolute difference between two successive $\mathrm{RR}_{n} \mathrm{I} / \mathrm{RR}_{n} \mathrm{I}_{m}$ sequences exceeds $50 \times n$ ms; pNN50n: NN50n divided by the total number of $\mathrm{RR}_{n} \mathrm{I} / \mathrm{RR}_{n} \mathrm{I}_{m}$ sequences; VLF: very low frequency; LF: low frequency; HF: high frequency; SD: standard deviation; SampEn: sample entropy; ApEn: approximate entropy; DFA: detrended fluctuation analysis. 
Table 3. Univariable analysis of HRV and HRnV parameters

\begin{tabular}{|c|c|c|c|c|c|c|}
\hline & HRV & & $\mathrm{HR}_{2} \mathrm{~V}$ & & $\mathrm{HR}_{3} \mathrm{~V}$ & \\
\hline & OR $(95 \%$ CI $)$ & $\mathbf{p}$ & OR $(95 \%$ CI $)$ & $\mathbf{P}$ & OR $(95 \%$ CI $)$ & $\mathbf{p}$ \\
\hline Mean NN & $1.000(0.998$ to 1.002$)$ & 0.903 & $1.000(0.999$ to 1.001$)$ & 0.964 & $1.000(0.999$ to 1.001$)$ & 0.978 \\
\hline SDNN & $1.021(1.008$ to 1.035$)$ & $0.002 *$ & $1.011(1.002$ to 1.020$)$ & $0.021 *$ & $1.007(1.000$ to 1.014$)$ & 0.065 \\
\hline RMSSD & 1.021 (1.009 to 1.032$)$ & $<0.001^{*}$ & 1.020 (1.008 to 1.032$)$ & $0.001 *$ & $1.019(1.007$ to 1.030$)$ & $0.002 *$ \\
\hline Skewness & $1.019(0.943$ to 1.102$)$ & 0.632 & $0.982(0.873$ to 1.105$)$ & 0.762 & $1.046(0.916$ to 1.193$)$ & 0.508 \\
\hline Kurtosis & 1.003 (0.999 to 1.007$)$ & 0.12 & 1.007 (0.999 to 1.015$)$ & 0.089 & $1.010(0.999$ to 1.020$)$ & 0.063 \\
\hline Triangular index & 1.044 (0.967 to 1.128$)$ & 0.271 & $1.029(0.970$ to 1.091$)$ & 0.342 & 1.014 (0.952 to 1.079$)$ & 0.668 \\
\hline NN50 & 1.004 (1.001 to 1.007$)$ & $0.016^{*}$ & 1.007 (1.001 to 1.014$)$ & $0.034 *$ & $1.010(1.000$ to 1.020$)$ & $0.048^{*}$ \\
\hline pNN50 & 1.024 (1.006 to 1.042$)$ & $0.008 *$ & $1.023(1.004$ to 1.041$)$ & $0.016^{*}$ & $1.019(1.002$ to 1.037$)$ & $0.032 *$ \\
\hline NN50n & - & - & 1.055 (1.013 to 1.099$)$ & $0.011^{*}$ & $1.087(1.023$ to 1.155$)$ & $0.007^{*}$ \\
\hline pNN50n & - & - & $1.122(1.025$ to 1.228$)$ & $0.013 *$ & $1.136(1.034$ to 1.248$)$ & $0.008 *$ \\
\hline Total power & $1.000(1.000$ to 1.000$)$ & $0.007 *$ & $1.000(1.000$ to 1.000$)$ & $0.015^{*}$ & 1.000 (1.000 to 1.000$)$ & 0.056 \\
\hline VLF power & $1.000(1.000$ to 1.001$)$ & 0.079 & 1.000 (1.000 to 1.000$)$ & 0.058 & 1.000 (1.000 to 1.000$)$ & $0.039^{*}$ \\
\hline LF power & 1.001 (1.000 to 1.001$)$ & $0.004 *$ & $1.000(1.000$ to 1.001$)$ & $0.021 *$ & $1.000(1.000$ to 1.001$)$ & $0.018^{*}$ \\
\hline HF power & 1.001 (1.000 to 1.001$)$ & $0.004 *$ & 1.001 (1.000 to 1.001$)$ & $0.001 *$ & $1.000(1.000$ to 1.000$)$ & 0.268 \\
\hline LF power norm & $0.978(0.962$ to 0.995$)$ & $0.009 *$ & $0.987(0.972$ to 1.002$)$ & 0.095 & $0.986(0.972$ to 1.001$)$ & 0.059 \\
\hline HF power norm & $1.022(1.005$ to 1.039$)$ & $0.009^{*}$ & 1.013 (0.998 to 1.029$)$ & 0.095 & 1.014 (0.999 to 1.029$)$ & 0.059 \\
\hline LF/HF & 0.682 (0.457 to 1.017$)$ & 0.061 & 0.856 (0.618 to 1.187$)$ & 0.352 & $0.960(0.810$ to 1.138$)$ & 0.637 \\
\hline Poincaré SD1 & 1.027 (1.010 to 1.044$)$ & $0.001 *$ & $1.028(1.011$ to 1.045$)$ & $0.001 *$ & 1.021 (1.007 to 1.037$)$ & $0.004 *$ \\
\hline Poincaré SD2 & 1.013 (1.002 to 1.024$)$ & $0.018^{*}$ & $1.007(1.000$ to 1.014$)$ & 0.066 & 1.003 (0.998 to 1.008$)$ & 0.235 \\
\hline Poincaré SD1/SD2 & $2.346(1.160$ to 4.745$)$ & $0.018^{*}$ & 8.160 (2.164 to 30.773$)$ & $0.002 *$ & 8.285 (2.541 to 27.017$)$ & $<0.001^{*}$ \\
\hline SampEn & $0.965(0.534$ to 1.741$)$ & 0.905 & $1.000(1.000$ to 1.000$)$ & 0.31 & $1.000(1.000$ to 1.000$)$ & 0.15 \\
\hline ApEn & $0.269(0.083$ to 0.879$)$ & $0.03^{*}$ & $0.261(0.079$ to 0.862$)$ & $0.028 *$ & $0.729(0.232$ to 2.288$)$ & 0.588 \\
\hline DFA, $\alpha 1$ & $0.704(0.243$ to 2.038$)$ & 0.518 & $0.342(0.123$ to 0.955$)$ & $0.041 *$ & $0.213(0.077$ to 0.590$)$ & $0.003 *$ \\
\hline \multirow[t]{3}{*}{ DFA, $\alpha 2$} & 0.137 (0.047 to 0.400$)$ & $<0.001 *$ & 0.352 (0.177 to 0.699$)$ & $0.003^{*}$ & 0.631 (0.433 to 0.918$)$ & $0.016^{*}$ \\
\hline & $\mathrm{HR}_{2} \mathrm{~V}_{1}$ & & $\mathrm{HR}_{3} \mathrm{~V}_{1}$ & & $\mathrm{HR}_{3} \mathrm{~V}_{2}$ & \\
\hline & OR (95\% CI) & $\mathbf{p}$ & OR (95\% CI) & $\mathbf{p}$ & OR (95\% CI) & $\mathbf{p}$ \\
\hline Mean NN & $1.000(0.999$ to 1.001$)$ & 0.905 & $1.000(0.999$ to 1.001$)$ & 0.906 & $1.000(0.999$ to 1.001$)$ & 0.965 \\
\hline SDNN & $1.010(1.003$ to 1.018$)$ & $0.008^{*}$ & 1.006 (1.001 to 1.012$)$ & $0.018^{*}$ & $1.007(1.000$ to 1.013$)$ & 0.053 \\
\hline RMSSD & 1.022 (1.011 to 1.034$)$ & $<0.001^{*}$ & 1.021 (1.010 to 1.033$)$ & $<0.001 *$ & 1.019 (1.007 to 1.032$)$ & $0.002 *$ \\
\hline Skewness & 0.934 (0.842 to 1.036$)$ & 0.198 & 0.909 (0.799 to 1.032$)$ & 0.141 & 0.954 (0.836 to 1.089$)$ & 0.485 \\
\hline Kurtosis & 1.007 (1.001 to 1.013$)$ & $0.022 *$ & 1.010 (1.001 to 1.018$)$ & $0.03^{*}$ & $1.009(0.999$ to 1.019$)$ & 0.092 \\
\hline Triangular index & 1.027 (0.972 to 1.085$)$ & 0.34 & 1.012 (0.969 to 1.057$)$ & 0.586 & 1.009 (0.954 to 1.066$)$ & 0.763 \\
\hline NN50 & 1.004 (1.001 to 1.007$)$ & $0.022 *$ & 1.004 (1.000 to 1.007$)$ & $0.034 *$ & 1.007 (1.000 to 1.014$)$ & $0.043^{*}$ \\
\hline pNN50 & 1.024 (1.006 to 1.042$)$ & $0.009^{*}$ & $1.022(1.004$ to 1.040$)$ & $0.017^{*}$ & 1.020 (1.002 to 1.038$)$ & $0.027 *$ \\
\hline NN50n & $1.026(1.006$ to 1.045$)$ & $0.009 *$ & 1.028 (1.007 to 1.049$)$ & $0.007 *$ & 1.064 (1.020 to 1.110$)$ & $0.004^{*}$ \\
\hline pNN50n & $1.120(1.028$ to 1.220$)$ & $0.01 *$ & 1.138 (1.036 to 1.249$)$ & $0.007 *$ & 1.151 (1.044 to 1.270$)$ & $0.005^{*}$ \\
\hline Total power & $1.000(1.000$ to 1.000$)$ & $0.015^{*}$ & 1.000 (1.000 to 1.000$)$ & $0.022^{*}$ & 1.000 (1.000 to 1.000$)$ & $0.031^{*}$ \\
\hline VLF power & $1.000(1.000$ to 1.000$)$ & 0.08 & $1.000(1.000$ to 1.000$)$ & 0.08 & 1.000 (1.000 to 1.000$)$ & 0.059 \\
\hline LF power & $1.000(1.000$ to 1.000$)$ & $0.005^{*}$ & 1.000 (1.000 to 1.000$)$ & $0.005^{*}$ & 1.000 (1.000 to 1.000$)$ & $0.026^{*}$ \\
\hline HF power & $1.000(1.000$ to 1.000$)$ & $0.002 *$ & $1.000(1.000$ to 1.000$)$ & $0.001 *$ & $1.001(1.000$ to 1.001$)$ & $0.001^{*}$ \\
\hline LF power norm & $0.980(0.965$ to 0.996$)$ & $0.013^{*}$ & 0.979 (0.965 to 0.994$)$ & $0.006 *$ & 0.979 (0.964 to 0.995$)$ & $0.009^{*}$ \\
\hline HF power norm & $1.020(1.004$ to 1.037$)$ & $0.013^{*}$ & 1.021 (1.006 to 1.037$)$ & $0.006^{*}$ & 1.021 (1.005 to 1.038$)$ & $0.009^{*}$ \\
\hline LF/HF & $0.769(0.570$ to 1.037$)$ & 0.085 & $0.883(0.744$ to 1.047$)$ & 0.153 & $0.884(0.752$ to 1.040$)$ & 0.137 \\
\hline Poincaré SD1 & 1.029 (1.012 to 1.047$)$ & $0.001 *$ & 1.028 (1.011 to 1.045$)$ & $0.001 *$ & 1.027 (1.010 to 1.044$)$ & $0.002^{*}$ \\
\hline Poincaré SD2 & $1.006(1.000$ to 1.011$)$ & $0.034 *$ & 1.004 (1.000 to 1.007$)$ & $0.048^{*}$ & 1.004 (0.999 to 1.009$)$ & 0.129 \\
\hline Poincaré SD1/SD2 & $7.893(2.085$ to 29.879$)$ & $0.002 *$ & 7.405 (1.867 to 29.365$)$ & $0.004 *$ & 7.610 (2.222 to 26.064$)$ & $0.001 *$ \\
\hline SampEn & $0.830(0.471$ to 1.461$)$ & 0.518 & 0.809 (0.480 to 1.364$)$ & 0.427 & 1.000 (1.000 to 1.000$)$ & 0.31 \\
\hline ApEn & 0.382 (0.122 to 1.192$)$ & 0.097 & $0.728(0.244$ to 2.170$)$ & 0.569 & 0.544 (0.175 to 1.693$)$ & 0.293 \\
\hline DFA, $\alpha 1$ & $0.480(0.171$ to 1.350$)$ & 0.164 & 0.471 (0.173 to 1.284$)$ & 0.141 & 0.380 (0.146 to 0.990$)$ & $0.048^{*}$ \\
\hline DFA, $\alpha 2$ & $0.141(0.047$ to 0.423$)$ & $<0.001 *$ & $0.146(0.048$ to 0.448$)$ & $0.001 *$ & $0.384(0.194$ to 0.760$)$ & $0.006^{*}$ \\
\hline
\end{tabular}

$* \mathrm{p}<0.05$. HRV: heart rate variability; OR: odds ratio; CI: confidence interval; mean NN: average of R-R intervals; SDNN: standard deviation of R-R intervals; RMSSD: square root of the mean squared differences between R-R intervals; NN50: the number of times that the absolute difference between two successive R-R intervals exceeds $50 \mathrm{~ms}$; pNN50: NN50 divided by the total number of R-R intervals; NN50n: the number of times that the absolute difference between 2 successive $\mathrm{RR}_{n} \mathrm{I} / \mathrm{RR}_{n} \mathrm{I}_{m}$ sequences exceeds $50 \times n$ ms; $\mathrm{pNN} 50 n$ : NN50n divided by the total number of $\mathrm{RR}_{n} \mathrm{I} / \mathrm{RR}_{n} \mathrm{I}_{m}$ sequences; VLF: very low frequency; LF: low frequency; HF: high frequency; SD: standard deviation; SampEn: sample entropy; ApEn: approximate entropy; DFA: detrended fluctuation analysis. 
medRxiv preprint doi: https://doi.org/10.1101/2020.12.26.20248866; this version posted January 2, 2021. The copyright holder for this preprint (which was not certified by peer review) is the author/funder, who has granted medRxiv a license to display the preprint in perpetuity.

It is made available under a CC-BY-NC-ND 4.0 International license .

Table 4: Multivariable analysis

\begin{tabular}{|c|c|c|}
\hline Variables & Adjusted Odds Ratio (95\% CI) & p-value \\
\hline \multicolumn{3}{|l|}{ Vital signs } \\
\hline Respiratory rate & $1.168(1.085$ to 1.257$)$ & $<0.001 *$ \\
\hline Systolic blood pressure & $0.978(0.966$ to 0.990$)$ & $0.001 *$ \\
\hline Glasgow coma scale & $0.845(0.769$ to 0.929$)$ & $0.001 *$ \\
\hline $\mathrm{SpO}_{2}$ & $0.892(0.838$ to 0.950$)$ & $<0.001 *$ \\
\hline \multicolumn{3}{|l|}{ HRV parameters } \\
\hline HRV total power & $1.000(1.000$ to 1.001$)$ & $0.019 *$ \\
\hline HRV Poincare SD1 & $0.948(0.893$ to 1.007$)$ & 0.081 \\
\hline \multicolumn{3}{|l|}{ HRnV parameters } \\
\hline $\mathrm{HR}_{2} \mathrm{~V}$ NN50 & $1.270(0.967$ to 1.667$)$ & 0.085 \\
\hline $\mathrm{HR}_{2} \mathrm{~V}$ HF power & $1.002(1.000$ to 1.003$)$ & 0.076 \\
\hline $\mathrm{HR}_{2} \mathrm{~V}$ pNN50 & $0.290(0.115$ to 0.732$)$ & $0.009^{*}$ \\
\hline $\mathrm{HR}_{2} \mathrm{~V}$ ApEn & $0.106(0.013$ to 0.877$)$ & $0.037 *$ \\
\hline $\mathrm{HR}_{2} \mathrm{~V}_{1} \mathrm{NN} 50$ & $0.808(0.682$ to 0.958$)$ & $0.014^{*}$ \\
\hline $\mathrm{HR}_{2} \mathrm{~V}_{1} \mathrm{pNN} 50$ & $5.700(1.784$ to 18.213$)$ & $0.003 *$ \\
\hline $\mathrm{HR}_{2} \mathrm{~V}_{1} \mathrm{LF} / \mathrm{HF}$ & $0.251(0.071$ to 0.880$)$ & $0.031 *$ \\
\hline $\mathrm{HR}_{3} \mathrm{~V}$ pNN50 & $1.229(0.988$ to 1.528$)$ & 0.064 \\
\hline $\mathrm{HR}_{3} \mathrm{~V}_{1} \mathrm{NN} 50$ & $1.098(0.999$ to 1.206$)$ & 0.053 \\
\hline $\mathrm{HR}_{3} \mathrm{~V}_{1} \mathrm{LF} / \mathrm{HF}$ & $2.241(1.252$ to 4.013$)$ & $0.007 *$ \\
\hline $\mathrm{HR}_{3} \mathrm{~V}_{1} \mathrm{pNN} 50$ & $0.473(0.247$ to 0.906$)$ & $0.024 *$ \\
\hline $\mathrm{HR}_{3} \mathrm{~V}_{1} \mathrm{NN} 50 n$ & $0.794(0.664$ to 0.950$)$ & $0.012 *$ \\
\hline $\mathrm{HR}_{3} \mathrm{~V}_{1} \mathrm{HF}$ power norm & $1.093(1.044$ to 1.144$)$ & $<0.001 *$ \\
\hline $\mathrm{HR}_{3} \mathrm{~V}_{1} \mathrm{DFA} \alpha 1$ & $14.189(1.009$ to 199.510$)$ & $0.049 *$ \\
\hline $\mathrm{HR}_{3} \mathrm{~V}_{2} \mathrm{NN} 50 n$ & $1.713(1.202$ to 2.443$)$ & $0.003 *$ \\
\hline
\end{tabular}

$* \mathrm{p}<0.05$

HRV: heart rate variability; CI: confidence interval; NN50: the number of times that the absolute difference between two successive R-R intervals exceeds $50 \mathrm{~ms}$; pNN50: NN50 divided by the total number of R-R intervals; NN50n: the number of times that the absolute difference between 2 successive $\mathrm{RR}_{n} \mathrm{I} / \mathrm{RR}_{n} \mathrm{I}_{m}$ sequences exceeds 50×n ms; LF: low frequency; HF: high frequency; SD: standard deviation; ApEn: approximate entropy; DFA: detrended fluctuation analysis. 


\section{Figures}

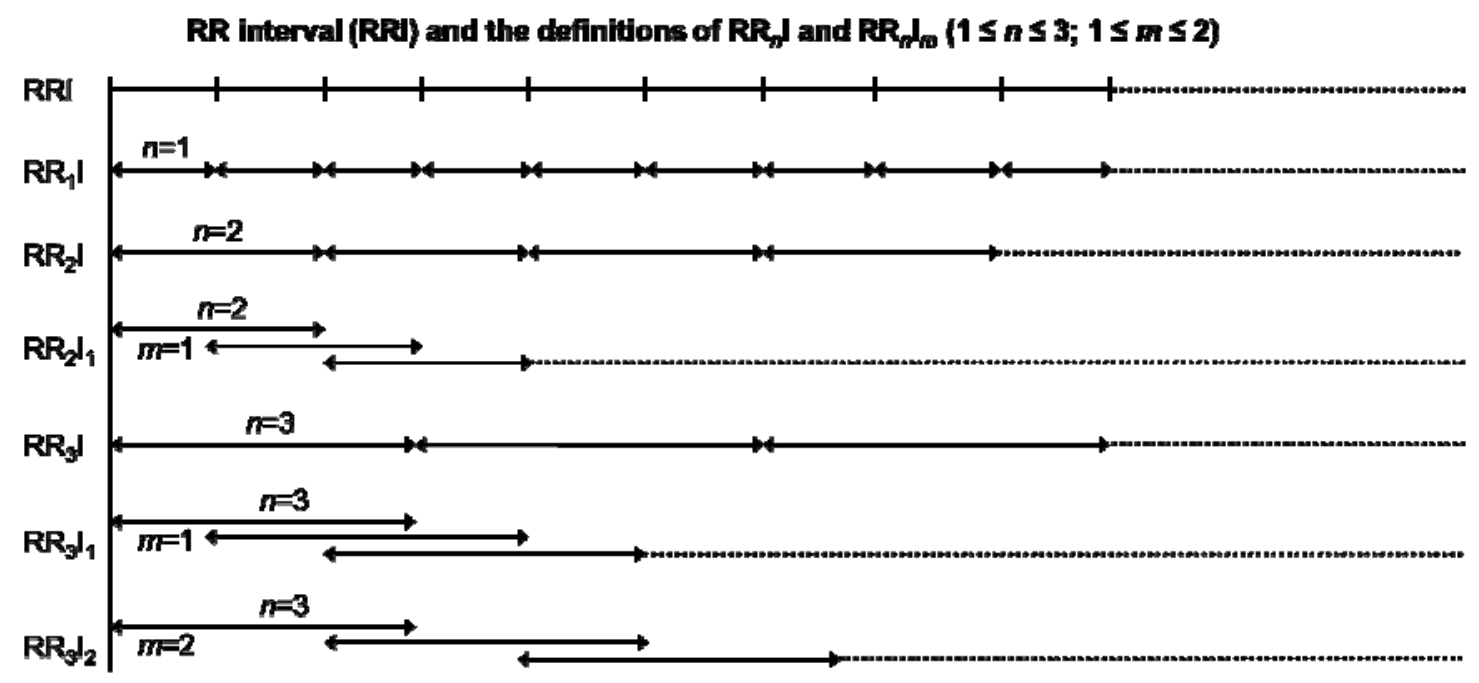

Figure 1. Illustration of the RR intervals (RRIs) and the definitions of $\mathrm{RR}_{n} \mathrm{I}$ and $\mathrm{RR}_{n} \mathrm{I}_{m}$, where $1 \leq n \leq 3,1 \leq m \leq 2$. Parameter $m$ indicates the non-overlapping portion between two successive $\mathrm{RR}_{n} \mathrm{I}_{m}$ sequences.

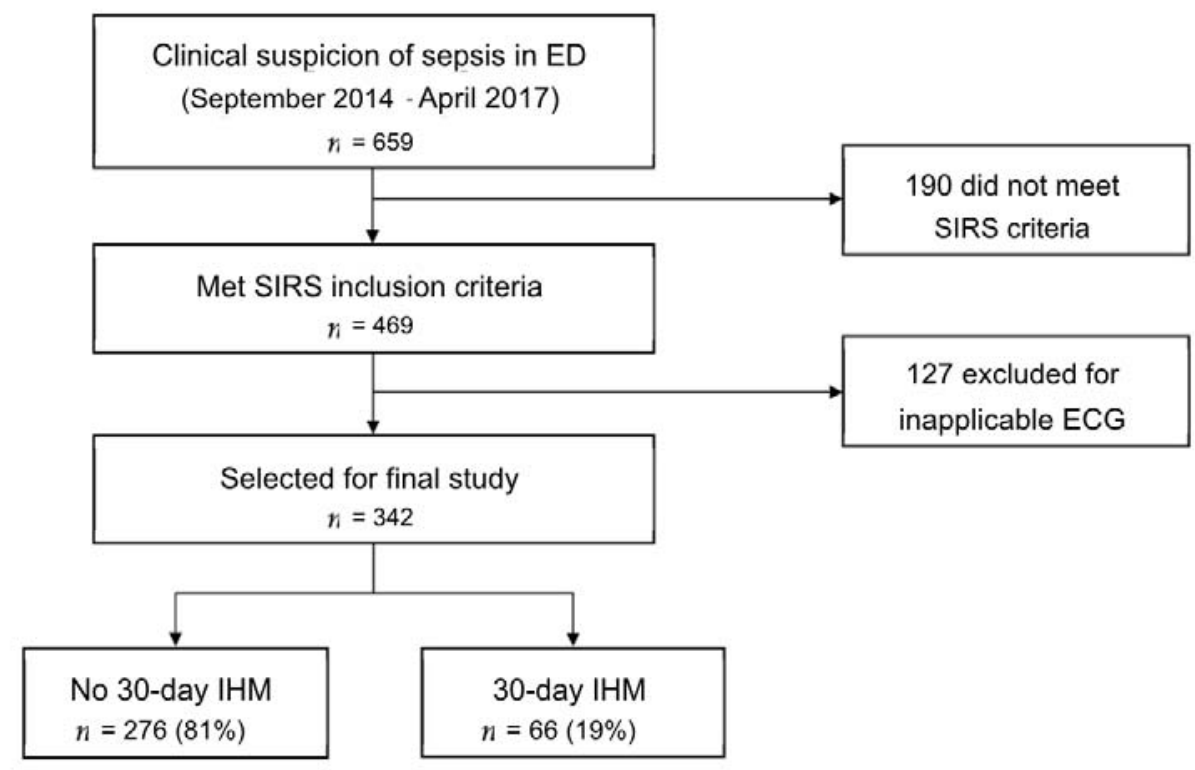

Figure 2. Patient recruitment flowchart. ECG: Electrocardiogram; ED: emergency department; IHM: in-hospital mortality; SIRS: systemic inflammatory response syndrome. 


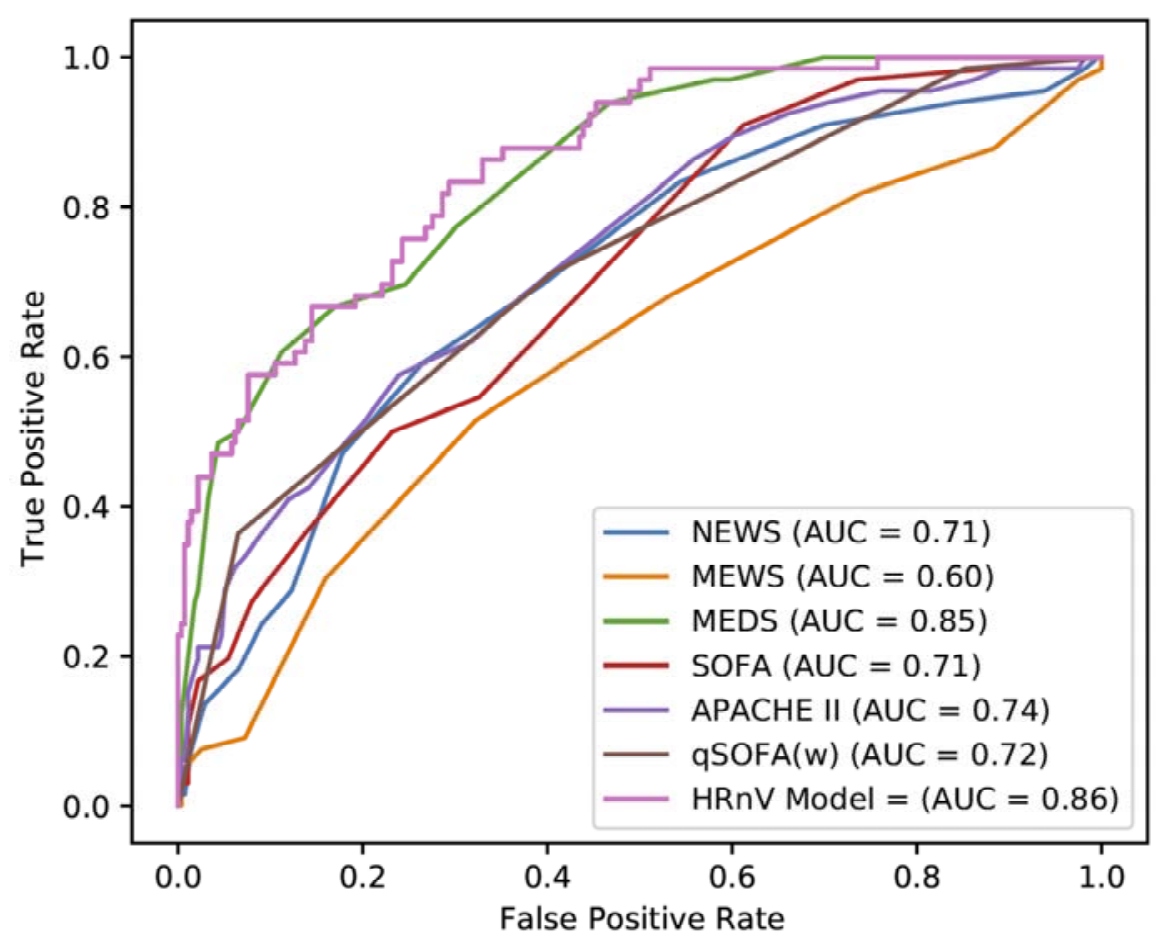

Figure 3. ROC curves for HRV and HRnV-based prediction models, alongside other disease severity scoring systems. Confidence intervals shown are for 95\%. APACHE II: Acute Physiology and Chronic Health Evaluation II; AUC: area under the receiver operating characteristic curve; HRnV: heart rate n-variability; HRV: heart rate variability; MEDS: Mortality in Emergency Department Sepsis; MEWS: Modified Early Warning Score; NEWS: National Early Warning Score; SOFA: Sequential Organ Failure Assessment; qSOFA(w): worst quick SOFA score. 